\title{
SDRA e ventilação
}

\author{
Guilherme P.P. SChettino ${ }^{1}$, J o Ão Batista Borges Sobrinho ${ }^{2}$
}

A última edição do Congresso da American Thoracic Society, realizada em San Diego, Califórnia - EUA, comprovou 0 avanço da produção científica nacional nas áreas relacionadas a insuficiência respiratória e ventilação mecânica. Foram apresentados 15 estudos, entre clínicos e experimentais, relacionados a diferentes linhas de pesquisa. Discutiremos a seguir como estes estudos se enquadram no contexto do conhecimento atual da insuficiência respiratória e ventilação mecânica.

Nós presentes a San Diego tivemos também o prazer de acompanhar várias discussões, nas sessões de Critical Care, NIH Conference e Clinical Year in Reviews, apreciando e reconhecendo a contribuição do estudo de Amato et al.(1) para a definição da estratégia ventilatória atual a ser empregada em pacientes com síndrome do desconforto respiratório agudo.

\section{I - SíndROME dO DESCONFORTO RESPIRATÓRIO AGUDO - VENTILAÇÃO MECÂNICA}

Desde sua descrição por Ashbaugh et al., em 1967(2), a síndrome do desconforto respiratório agudo (SDRA) tem-se constituído em um foco intenso de pesquisas clínicas e experimentais. Contudo, apesar dos avanços nas medidas de suporte, a ocorrência desta síndrome é um importante determinante de morbidade e mortalidade nas unidades de terapia intensiva ${ }^{(3)}$.

A SDRA é uma entidade clínica decorrente de vários insultos locais ou sistêmicos que acarretam lesão da estrutura alvéolo-capilar, com aumento da permeabilidade capilar. Inflamação aguda e perda da função de barreira resultam em edema exsudativo e distúrbios quantitativos e qualitativos do surfactante da via aérea terminal e do espaço alveolar. O corre aumento do peso do pulmão, colapso heterogêneo dos espaços aéreos - predominantemente nas regiões dependentes - e alterações graves da troca gasosa. Clinicamente, ela é definida pela presença de infiltrado pulmonar agudo, diminuição da complacência pulmonar e hipoxemia grave (relação $\left.\mathrm{PaO}_{2} / \mathrm{FiO}_{2}<200 \mathrm{mmHg}\right)^{(3)}$.

Vários mediadores inflamatórios são encontrados no espaço alveolar, mesmo na fase inicial da SDRA, incluindo

1. Presidente da Comissão de Terapia Intensiva da SBPT; Médico Assistente UTI Respiratória, Divisão de Doenças Respiratórias-Incor/HC/FMUSP; E-mail: ials@opus.com.br

2. Médico pós-graduando, Divisão de Doenças Respiratórias-Incor/ HC/ FMUSP. enzimas que podem desempenhar um papel importante no remodelamento pulmonar. Durante a lesão pulmonar aguda a barreira endotelial e a epitelial são lesadas e a compartimentalização das citocinas alveolares pode ser perdida, com seu "escape" para o interior do espaço vascular, podendo iniciar ou propagar uma resposta inflamatória sistêmica(4-6). O pulmão poderia assim ser um dos responsáveis pelo resposta inflamatória sistêmica presente nesta síndrome e não somente um órgão-alvo.

Em muitos pacientes, a síntese de colágeno, e o conseqüente desenvolvimento de lesões fibróticas, inicia-se precocemente ainda durante a fase aguda da doença(7). Estudos recentes procuram inclusive diferenciar os dados de mecânica pulmonar e de reação inflamatória da SDRA primária (lesão primária pulmonar) e SDRA secundária (lesão pulmonar conseqüente a processo inflamatório extrapulmonar).

Existem evidências atuais demonstrando que a estratégia usada na ventilação mecânica pode afetar a atividade inflamatória pulmonar durante a SDRA, assim como a resposta inflamatória sistêmica(8-13). Baseados nestes dados, é indispensável avançar o conhecimento acerca do potencial protetor ou lesivo da ventilação mecânica.

Nos últimos anos ocorreu uma transformação nas estratégias ventilatórias utilizadas nos pacientes com SDRA: de uma abordagem inicial na qual os ventiladores mecânicos eram ajustados buscando-se parâmetros de mecânica respiratória e de troca gasosa os mais próximos possíveis dos dados fisiológicos encontrados em pulmões normais, para um conceito atual em que o objetivo principal é evitar os efeitos adversos do suporte ventilatório enquanto se tenta fornecer a melhor ventilação possível|(14). Esta mudança de paradigma é baseada no crescente entendimento de que a ventilação mecânica pode piorar uma lesão pulmonar preexistente, ou pode produzir lesão pulmonar aguda de novo em pulmões previamente saudáveis(15,16).

Pressões intersticiais negativas ${ }^{(17-21)}$, criadas e amplificadas durante a distensão do espaço alveolar, são responsabilizadas por grandes pressões transmurais nos capilares pulmonares, justificando vários efeitos deletérios associados à ventilação mecânica(22-26). West e colaboradores demonstraram que a membrana basal e 0 endotélio dos capilares pulmonares podem sofrer enormes tensões radiais e rupturas quando o pulmão é submetido a grandes variações volumétricas, especialmente na presença de elevadas tensões superficiais (ex.: deficiência de surfactante, presente na SDRA) (24, 26-28). 
Pressões inspiratórias comumente empregadas na prática clínica, como, por exemplo, pressão na via aérea maior que $30 \mathrm{cmH}_{2} \mathrm{O}$, quando utilizadas por período tão curto como 20 minutos, produzem efeitos deletérios em pulmões de ratos, coelhos, cães, porcos e ovelhas, com evidente lesão e desorganização do interstício pulmonar ${ }^{(16,21,23,29-38)}$. Ao mesmo tempo, uma série de experimentos alertaram que as preocupações não deveriam concentrar-se apenas nos efeitos da hiperdistensão pulmonar. A presença de colapso alveolar, quer seja cíclico (apenas ao final da expiração), quer seja durante todo o ciclo respiratório, passou a ser considerado como um potencializador das lesões durante a ventilação mecânica(23,29,31,36,39-41). Mead e colaboradores demonstraram que a interface entre zonas colapsadas e aeradas poderia sofrer a ação de grandes forças de cisalhamento, com fratura capilar durante a ventilação alveolar ${ }^{(42,43)}$. Neste modelo geométrico do tecido pulmonar, onde há a interdependência elástica das paredes alveolares, a parede de um alvéolo colapsado envolto por unidades abertas pode receber uma tensão equivalente a $140 \mathrm{cmH}_{2} \mathrm{O}$ durante uma insuflação usual com $30 \mathrm{CmH}_{2} \mathrm{O}$.

Estudos com modelos de deficiência de surfactante demonstraram que colapsos cíclicos, mesmo que restritos às zonas dos bronquíolos terminais, poderiam também gerar grandes desarranjos. De acordo com recentes evidências, quando paredes opostas de um bronquíolo terminal se aproximam e "colam" sob efeito da alta tensão superficial do líquido em seu interior, sua reabertura requer altas pressões (pressão crítica de abertura)(21,44-46).

Trabalhos concomitantes demonstraram que a prevenção do colapso alveolar e bronquiolar - através de recrutamento alveolar e emprego da PEEP evitando o "colamento" das paredes bronquiolares e prevenindo a criação das perigosas interfaces entre regiões aeradas/ colapsadas - poderia atenuar o desenvolvimento destes processos lesivos em modelos experimentais e mesmo em estudos clínicos ${ }^{(36,39,41,48-50)}$.

Q uando to das as evidências científicas são consideradas, a reversão do colapso pulmonar emerge como algo crucial para uma estratégia protetora para os pulmões durante a ventilação mecânica na SDRA (51).

Podemos resumir estes conceitos em poucas palavras: devese usar a ventilação mecânica de modo a abrir os alvéolos e mantê-los abertos, e minimizando-se ao máximo a hiperdistensão pulmonar.

U m número crescente de autores tem advogado a idéia do uso da PEEP acima do ponto de inflexão $\left(P_{F L E X}\right)$ inferior da curva pressão-volume $(\mathrm{P}-\mathrm{V})$ para minimizar a lesão induzida pela ventilação mecânica provocada pela reabertura e fechamento cíclicos dos alvéolos ${ }^{(52)}$. A lém disso, têm-se buscado estratégias que evitem a hiperdistensão pulmonar, através da redução do volume corrente. São tentativas de respeitar o compromisso supracitado.
Recentemente, demonstrou-se pela primeira vez que uma estratégia protetora de ventilação mecânica é capaz de reduzir a mortalidade da SDRA quando comparada com uma estratégia convencional(1). Nesta estratégia ventilatória protetora, Amato et al. ajustaram o nível da PEEP $2 \mathrm{cmH}_{2} \mathrm{O}$ acima do $\mathrm{P}_{\mathrm{FLEX}}$ inferior da curva $\mathrm{P}-\mathrm{V}$, mantiveram a pressão de distensão $\left(\mathrm{P}_{\text {PLAT }}-\mathrm{PEEP}\right)$ menor que $20 \mathrm{cmH}_{2} \mathrm{O}$, e reduziram 0 volume corrente para menos de $6 \mathrm{ml} / \mathrm{kg}^{(64)}$.

O bservações mais recentes têm sugerido, contudo, que ainda podemos e devemos avançar no alcance de um ajuste ótimo de parâmetros mecânicos, ou no alcance de uma combinação ótima da PEEP e relações $P$-V. Isto porque sabemos que 0 uso de PEEP acima do $P_{F L E X}$ inferior pode não assegurar o recrutamento pulmonar máximo ou quase-máximo. Existem evidências de que, mesmo depois de uma manobra de recrutamento com altas pressões, a PEEP necessária para manter o pulmão humano aberto pode ser maior que o $P_{F L E X}$ inferior da curva P-V inspiratória(53). A lém disso, o estudo do componente descendente da relação P-V (deflação ou curva expiratória) mostra que os pulmões encontram-se num grau de recrutamento maior nesta fase do que na fase ascendente (inspiratória) da curva ${ }^{(53)}$. As alças expiratórias da relação P$V$ sofrem uma determinante influência do grau de recrutamento alcançado ao fim da alça inspiratória. N este contexto, são fundamentais estudos que comparem e que apontem quais são as manobras de recrutamento capazes de maximamente recrutar o pulmão, bem como nos forneçam informações claras sobre a segurança das mesmas. 0 próprio papel deste recrutamento máximo, ou quase-máximo, na evolução da SDRA, ainda merece maior elucidação.

Esta possibilidade, qual seja, a de explorar o ramo expiratório da curva $P-V$, permite a identificação de pressões críticas, abaixo das quais a perda do recrutamento pulmonar é evidente. Estas pressões parecem ser, em muitos casos, maiores do que as pressões relacionadas à "PEEP ótima" da etapa incremental (inspiratória) - definida como PEEP relacionada com a melhor complacência - indicando que um ajuste de PEEP nestes níveis (PEEP ótima da etapa inspiratória) não garante um recrutamento completo por tempo prolongado.

As distribuições das pressões de abertura e de fechamento (colapso) em pacientes com SDRA, derivadas da relação P$V$, têm sido alvo crescente de estudo, inclusive de novas tentativas de modelagem matemática. Há um inequívoco avanço na capacidade destas equações em descrever o comportamento obtido de pacientes com SDRA, mas está claro que ainda não temos uma solução matemática precisa, que alcance todas as possibilidades da complexa relação P-V na SDRA. Este progresso, desde que foram reconhecidas as importantes implicações da diferença entre as pressões de abertura e de fechamento(54), foi justamente impulsionado pela obtenção e tentativa crescentes da melhor maneira de fazer e interpretar a curva P-V de pulmões com lesão pulmo- 
nar aguda e SDRA. Porém, ainda não esgotamos o potencial da curva P-V, nem delimitamos com a exatidão necessária suas limitações.

Dúvidas - como ficou evidente em recente editorial da Critical Care Medicine ${ }^{(56)}$ - com menor ou maior pertinência, sobre se a PEEP tem um efeito somente cosmético ou verdadeiramente terapêutico na SDRA ainda perduram por mais de 30 anos (tempo em que a PEEP tem sido usada em pacientes com SDRA), sinal de que muito ainda temos que esclarecer e acrescentar ao conjunto dos conhecimentos a respeito desta doença, e sobre o papel exato de antigas e novas medidas terapêuticas.

MORPHOLOGICAL CHANGES OF CAROTID BODIES IN ACUTE RESPIRATORY INSUFFICIENCY: A LIGHT MICROSCOPY MORPHOMETRIC STUDY IN HUMANS. Vinhaes et al. - Este estudo apresenta os dados quantitativos da análise histológica (morfometria para avaliação do volume proporcional das células claras, escuras, sustentação e progenitoras) do corpo carotídeo (obtidos da realização de necropsia) de pacientes com os seguintes diagnósticos: síndrome do desconforto respiratório agudo - ARDS $(n=7)$, hipertensão arterial sistêmica $(n=$ 7), DPOC ( $n=8)$, controle $(n=9)$. Pacientes com ARDS apresentaram aumento significativo do volume proporcional das células escuras quando comparados com o restante dos grupos, enquanto aqueles com DPOC apresentaram aumento das células de sustentação e redução do tecido glômico quando comparados com o grupo-controle. Os autores chamam a atenção para a necessidade de estudos para o avanço do entendimento do controle da respiração na hipoxemia aguda. A poio: CNPq, FAPESP, LIN05-HC/FMUSP.

Pressure-Volume curves in ARDS: COMPARISON OF TWO METHODS. Meyer et al. - Este estudo foi feito para determinar a correlação entre duas diferentes técnicas de aquisição da curva P-V, a técnica do fluxo contínuo e a técnica da oclusão rápida de via aérea ao final da inspiração, para obtenção do $P_{F L E X}$ inferior e do $P_{F L E X}$ superior da curva P-V de 13 pacientes com SDRA. A técnica do fluxo contínuo foi feita com um fluxo de $1 \mathrm{~L} / \mathrm{min}$, depois de um CPAP de $40 \mathrm{cmH}_{2} \mathrm{O}$ seguido de uma desconexão; na técnica da oclusão rápida de via aérea ao final da inspiração, diferentes volumes correntes randomizados foram testados depois de uma homogeneização da história pulmonar. Os resultados demonstraram que 0 $P_{F L E X}$ inferior pode ser encontrado na maioria dos pacientes, e que o $P_{F L E X}$ inferior e o $P_{F L E X}$ superior da curva $P-V$ são maiores quando obtidos com a técnica do fluxo contínuo. Os autores acreditam que a diferença observada entre os dois métodos pode ser atribuída a diferenças na história pulmonar e no recrutamento durante o procedimento da técnica da oclusão rápida de via aérea ao final da inspiração. Apoio: FA. PESP-Pronex.

TIDAL RECRUITMENT MISINTERPRETED AS VISCOELASTIC PRESSURE DISSIPATION IN ARDS PATIENTS. Grunauer et al. - A proposta deste estudo foi analisar a dissipação viscoelástica de pressões no sistema respiratório em condições de recrutamento pulmonar progressivo e subseqüente perda progressiva do mesmo. Foram estudados seis pacientes com SDRA, nos quais passos crescentes e decrescentes de PEEP foram subseqüentemente aplicados, e a pressão resultante da subtração da pressão de platô da pressão no momento de fluxo zero foi calculada em cada nível de PEEP. Condições de recrutamento progressivo, e perda progressiva do mesmo parecem ter sido responsáveis pelas diferenças na dissipação de pressões viscoelásticas entre passos crescentes e decrescentes de PEEP. Os resultados sugerem que o fenômeno da adaptação ao estresse "clássico" do tecido pulmonar pode depender de alterações da área de superfície pulmonar durante a pausa inspiratória. Apoio: FAPESP, LIM-HC/FMUSP.

DIFFERENT CONSTANT FLOWS CAN EQUALLY DETERMINE THE LOWER INFLECTION POINT IN ARDS PATIENTS. Gama et al. Para avaliar a influência de quatro diferentes fluxos contínuos $\left(1,2,5\right.$ e 10L/ min) na determinação do $P_{F L E X}$ inferior da curva P-V, foram estudados sete pacientes com SDRA. Depois de uma manobra de CPAP de $40 \mathrm{cmH}_{2} \mathrm{O}$ por 15 segundos, e 10 segundos de exalação para a atmosfera, os sete pacientes receberam, numa ordem randomizada, os quatro fluxos contínuos. Como resultado, não houve diferença entre os fluxos ao se procurar identificar o $\mathrm{P}_{\mathrm{FLEX}}$ inferior. Os autores concluem que os fluxos contínuos de 1 a 10L/min podem igualmente determinar o $\mathrm{P}_{\mathrm{FLEX}}$ inferior da curva $\mathrm{P}-\mathrm{V}$ de pacientes com SDRA. Apoio: FAPESP, LIM-HC/FMUSP.

PEEP AFTER A RECRUITMENT MANEUVER CAN KEEP A GREATER MEAN ALVEOLAR DIAMETER IN VALI RATS. Mori et al. - Os efeitos da PEEP na morfometria pulmonar antes e depois de uma manobra de recrutamento, de acordo com uma curva P. $V$, na lesão pulmonar associada ao ventilador em ratos não são bem conhecidos. Foram estudados 48 ratos randomizados em oito grupos, depois de três curvas P-V semi-estáticas (com um fluxo contínuo de $1,66 \mathrm{~L} / \mathrm{min}$ ) terem sido feitas. A lesão pulmonar associada ao ventilador foi induzida ventilando os ratos com volume corrente de $45 \mathrm{~mL} / \mathrm{kg} \mathrm{e} 5 \mathrm{cmH}_{2} \mathrm{O}$ de PEEP, até que se observasse um incremento de $30 \%$ da elastância do sistema respiratório. A través do uso da morfometria, mostrou-se que a lesão pulmonar associada ao ventilador é caracterizada histologicamente por hemorragia. E o resultado principal foi que a fixação no mesmo CPAP de $2 \mathrm{cmH}_{2} \mathrm{O}$ abaixo do $\mathrm{P}_{\mathrm{FLEX}}$ inferior da curva P-V, depois de uma manobra de recrutamento, levou a um maior diâmetro alveolar médio em ratos com lesão pulmonar associada ao ventilador. Apoio: FAPESP, LIM-HC/FMUSP.

Finding the "best PEEP" in ARDS patients: influence OF TIDAL VOLUME AND LUNG RECRUITMENT IN THE ESTIMATION OF THE BEST COMPLIANCE. Meyer et al. - Seis pacientes com SDRA foram avaliados para determinar a influência do volume corrente e do recrutamento pulmonar (a cada nível de 
PEEP) na complacência estática do sistema respiratório. Foi feita uma manobra ascendente e descendente, passo a passo, com volume corrente $\left(\mathrm{V}_{\mathrm{T}}\right)$ alto e volume corrente baixo (10 e $4 \mathrm{ml} / \mathrm{kg}$, respectivamente): passos de PEEP de $2 \mathrm{cmH}_{2} \mathrm{O}$ foram aplicados até uma pressão de platô de $55 \mathrm{cmH}_{2} \mathrm{O}$, e então ele foi progressivamente removido. A "PEEP ótima" foi definida como a PEEP relacionada à melhor complacência. A "PEEP ótima" resultou maior na fase descendente que na ascendente da curva PEEP-complacência que mediu a complacência estática nos níveis crescentes e decrescentes de PEEP. Tal achado sugeriu para os autores que, depois de uma manobra de recrutamento a pressão, um dado valor de PEEP obtém um volume pulmonar expiratório final maior. A lém disso, conforme os outros resultados e a interpretação dos autores, o uso do $\mathrm{V}_{\mathrm{T}}$ menor permitiu um recrutamento pulmonar maior a um mesmo limite de pressão, enquanto que o $V_{T}$ maior, ao causar tidal recruitment, subestimou a "PEEP ótima". Apoio: FAPESP, PRONEX, LIM-HC/FMUSP.

EFFECTS OF FUROSEMIDE IN RESPIRATORY MUCUS PROPERTIES AND TRANSPORTABILITY IN PATIENTS UNDER MECHANICAL VENTILATION. Kondo et al. - Os autores investigaram as propriedades físicas e a transportabilidade do muco de 27 pacientes submetidos a ventilação mecânica - amostras de muco colhidas nos tempos $0,1,2$, 3 e 4 horas -, divididos em grupo furosemida ( $n=12$, representando aqueles que receberam diurético, 10 a $200 \mathrm{mg})$ e controle $(n=15)$. 0 administração do diurético alterou significativamente o transporte do muco nesta população. Os pesquisadores discutem que as alterações encontradas podem ser devidas à ação direta da furosemida na mucosa das vias aéreas e/ ou pela mudança da volemia dos pacientes. Apoio: FAPESP, CNPq.

REPETITIVE HIGH PRESSURE RECRUITMENT MANEUVERS REQUIRED TO MAXIMALLY RECRUIT LUNG IN ARDS SHEEP MODEL. Fujino et al. - Foi comparada a efetividade de duas manobras elaboradas para recrutar maximamente o pulmão, num modelo animal de SDRA feito com lavagem do pulmão com solução salina. Uma consistia de CPAP de $40 \mathrm{cmH}_{2} \mathrm{O}$ por um minuto, e a outra de ventilação com pressão controlada de $20 \mathrm{cmH}_{2} \mathrm{O}$ com PEEP de $40 \mathrm{cmH}_{2} \mathrm{O}$ por dois minutos. $C$ ada manobra de recrutamento foi repetida quatro vezes. $\mathrm{A}$ oxigenação melhorou significativamente no grupo da ventilação com pressão controlada de $20 \mathrm{cmH}_{2} \mathrm{O}$ com PEEP de $40 \mathrm{cmH}_{2} \mathrm{O}$ depois da segunda manobra de recrutamento. Os autores concluem que manobras de recrutamento repetitivas, com pressões relativamente altas de via aérea, são necessárias para maximamente recrutar o pulmão colapsado na SDRA. A poio: Departmental Funds.

MANNITOL PARTIALLY PREVENTS ACUTE LUNG INJURY INDUCED BY OLEIC ACID IN RATS. Hilkner-Silva et al. - A infusão de ácido oléico é um modelo experimental clássico de lesão pulmonar aguda. Os autores estudaram os efeitos da infusão de manitol nas alterações pulmonares agudas em ratos nos quais foi infundido ácido oléico. Eles infundiram manitol 15 minutos antes ou duas horas depois da infusão do ácido oléico. Foram medidos a quantidade de edema alveolar e a heterogeneidade respiratória (colapso alveolar + hiperdistensão) quatro horas depois da infusão de ácido oléico, por análise morfométrica. Baseados nos resultados obtidos, os autores concluem que a análise morfométrica é um bom método para a detecção de lesões pulmonares em lesão pulmonar aguda induzida por ácido oléico, tão precoce quanto quatro horas depois da infusão de ácido oléico. A infusão de manitol parcialmente preveniu esta lesão pulmonar aguda. Apoio: CNPq, FAPESP, PRONEX-MCT.

EXTRACELLULAR MATRIX (ECM) REMODELING AND LUNG MECHANICS IN EARLY AND LATE ARDS: DIFFERENCES BETWEEN PULMONARY AND EXTRA-PULMONARY DISEASE. Negri et al. Com o objetivo de avaliar as alterações na matriz extracelular na SDRA, 37 pacientes com SDRA foram retrospectivamente estudados e divididos em grupos de acordo com a fase (doença precoce ou tardia) e a etiologia da SDRA: pulmonar (pneumonia, por exemplo) ou extrapulmonar (sepse, circulação extracorpórea, por exemplo). U sando análise por imagem, o conteúdo de fibras elásticas e de colágeno do septo alveolar foi quantificado em amostras coradas de pulmão autopsiado. A lém disso, valores de complacência dinâmica do sistema respiratório no dia da morte foram retrospectivamente acessados. $\mathrm{Na}$ fase precoce da SDRA o conteúdo de fibras de colágeno foi significativamente maior no grupo de etiologia pulmonar, enquanto que na fase tardia nenhuma diferença foi observada no conteúdo de fibras entre os grupos de doença. A complacência dinâmica foi sempre maior na SDRA extrapulmonar nas fases precoce e tardia. Os autores concluem que o remodelamento pulmonar na SDRA parece ser influenciado pelo sítio inicial da lesão (pulmonar ou extrapulmonar), e que as propriedades mecânicas do parênquima pulmonar podem ser influenciadas pelas diferenças. Apoio: CNPq, FAPESP, LIM-HC/ FMUSP, CAPES.

OXYGENATION AND MECHANICS PROFILE BEFORE AND AFTER CARDIOPULMONARY BYPASS. Crespo et al. - Estudo avalia os dados de trocas gasosas (relação $\mathrm{PaO}_{2} / \mathrm{FiO}_{2}$ ) e a mecânica ventilatória (complacência estática e resistência ao fluxo aéreo do sistema respiratório) de 24 pacientes antes e após 0 uso de circulação extracorpórea (tempo médio $=108,3 \mathrm{mi}$ nutos). O bservou-se queda da $\mathrm{PaO}_{2} / \mathrm{FiO}_{2}(417,52+124,15$ para $320,08+100,71$ ) não havendo, entretanto, alteração dos parâmetros de mecânica ventilatória estudados.

DISTRIBUTIONS OF OPENING AND CLOSING PRESSURES IN ARDS PATIENTS DERIVED FROM THE PRESSURE-VOLUME (PV) RELATIONSHIP. Venegas et al. - U ma equação empírica prévia ajusta dados da relação P-V de humanos e de animais extremamente bem. Nesta equação uma função dada aproxima a integral de uma distribuição normal, podendo representar o progressivo recrutamento dos alvéolos, ou a pro- 
gressiva perda do mesmo, com pressões de abertura ou de fechamento, respectivamente, distribuídas normalmente. Os autores multiplicaram tal função de "recrutamento fracional" pela "função elástica" exponencial clássica, e testaram este ajuste com as curvas P-V inspiratórias e expiratórias (obtidas pelo método da superseringa) de oito pacientes com SDRA. A nova equação descreveu os dados com alta acurácia, inclusive dando suporte - segundo os autores - ao conceito de baby lung em SDRA.

\section{II - Ventilação não INVASIVA COM PRESSÃo POSitiva, SINCRONIA PACIENTE - VENTILADOR E AUTO-PEEP}

A ventilação mecânica é utilizada na insuficiência respiratória com quatro objetivos principais: manutenção das trocas gasosas pulmonares (correção da hipoxemia e garantia da ventilação alveolar para eliminação do $\mathrm{CO}_{2}$ ); diminuição do trabalho respiratório (prevenção ou tratamento da fadiga muscular); manutenção dos volumes pulmonares (prevenção ou correção do colapso alveolar); diminuição da dispnéia (conforto).

Classicamente esses objetivos são alcançados com a aplicação da ventilação mecânica pulmonar através de uma prótese traqueal. Porém, hoje sabemos que os mesmos objetivos também podem ser alcançados, em pacientes selecionados, com o uso da ventilação não invasiva com pressão positiva (V NI), sem os riscos inerentes ao uso do tubo traqueal.

Vários modos de ventilação mecânica com pressão positiva, quando aplicados pela técnica não invasiva, foram eficientes em diminuir a necessidade de intubação traqueal e promover a melhora das trocas gasosas em pacientes graves. Entretanto, dúvidas persistem acerca do melhor modo a ser utilizado. Basicamente, a discussão gira em torno das vantagens e desvantagens dos modos limitados à pressão com o fluxo livre (por exemplo: CPAP, pressão de suporte $P S$, pressão controlada - PC, ventilação espontânea com dois níveis de pressão positiva - BiPAP) quando comparados com aqueles modos ciclados a volume com fluxo constante (por exemplo: volume assistido-controlado - Va/ C). Os modos volume garantido na pressão de suporte (VAPSV) $)^{(57-59)} \mathrm{e}$ principalmente ventilação assistida proporcional (Proportional assist ventilation - PAV) promovendo uma maior sincronia paciente-ventilador têm também se mostrado promissores durante a VNI(60). Entretanto, poucos autores compararam diretamente diferentes modos durante a VNI. Vitacca et al. ${ }^{(61)}$ demonstraram que o sucesso em evitar a intubação foi semelhante quando utilizaram PS ou Va/ c $(87,5 \%$ e $77 \%$ respectivamente) administrados por uma máscara facial em 29 pacientes consecutivos com doença pulmonar obstrutiva crônica agudizada. Posteriormente, A ppendini et al. ${ }^{(62)}$ demonstraram o sinergismo dos efeitos benéficos da PS e da CPAP na mecânica ventilatória, principalmente causando redução do trabalho respiratório, em pacientes obstrutivos. Girault et al. ${ }^{(63)}$ observaram que tanto do uso de PS quando $\mathrm{Va} / \mathrm{c}$ são efetivos em diminuir o trabalho respiratório em paciente com DPOC agudizada, porém com maior conforto

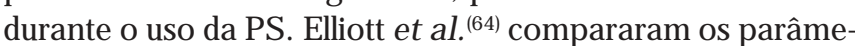
tros de mecânica respiratória e trocas gasosas de 11 pacientes com insuficiência respiratória com hipercapnia ventilados com uma máscara nasal nos modos Va/C, PS, PS + PEEP e CPAP. Os resultados obtidos durante o uso dos três primeiros modos foram semelhantes e superiores ao CPAP. Mehta et al. ${ }^{(65)}$ compararam 0 uso da PS e CPAP em 27 pacientes com insuficiência respiratória por edema pulmonar cardiogênico. A PS foi mais eficaz em corrigir as trocas gasosas e em oferecer conforto, porém, a taxa de infarto agudo do miocárdio foi surpreendente e significativamente maior nos pacientes do grupo da PS do que naqueles do grupo da CPAP, mesmo quando comparada com valores de uma série histórica $(71 \%, 31 \%$ e $38 \%$ respectivamente). Gay et al. ${ }^{(60)}$ demonstraram recentemente que o modo PAV é capaz de diminuir o trabalho respiratório de forma semeIhante à PS em pacientes com DPOC agudizada, porém oferecendo uma melhor sincronia paciente-ventilador e conforto. Calderini et al. ${ }^{(66)}$ demonstraram que 0 modo pressão controlada (pressão controlada com ciclos assistidos) propicia melhor sincronia paciente-ventilador quando comparado com o modo PS na presença de vazamento de ar ao redor da máscara, fato próprio da VNI.

O s resultados desses e de vários outros estudos não foram suficientes para responder quais os parâmetros ventilatórios e de trocas gasosas, e qual o peso destes, isoladamente ou em conjunto, realmente são determinantes para o conforto e sucesso da VNI. Acreditamos que a escolha do modo, assim como ajuste de seus parâmetros ventilatórios, deva ser feita de forma individualizada e baseada nas alterações fisiopatológicas encontradas.

FLOW PATTERNS IN NORMAL SUBJ ECTS UNDER PRESSURE SUPPORT VENTILATION AT DIFFERENT SLOPES AND INSPIRATORY RESISTANCE. Borges et al. - Este experimento realizado com voluntários, entretanto simulando também obstrução de vias aéreas, discute de forma elaborada aspectos relacionados à sincronia paciente-ventilator durante o uso do modo pressão de suporte - $\mathrm{PS} \pm 8 \mathrm{cmH}_{2} \mathrm{O}$ administrado por uma máscara facial - principalmente a influência de diferentes ajustes do flow rate na mecânica ventilatória. Os resultados demonstram que o pico de fluxo inspiratório e o tempo necessário para alcançar o pico de fluxo inspiratório variam de forma significante dependendo do acerto do flow rate, sem que houvesse variação do volume corrente ou tempo inspiratório. Os autores acreditam que a busca do melhor ajuste da PS pode ser útil em melhorar o conforto e, conseqüentemente, a aceitação deste modo, inclusive durante a ventilação não invasiva. Apoio: FAPESP, LIM-HC/FMUSP. 
Ventilatory mechanics PatTerns duRING the USE OF AN INTRINSIC PEEP SIMULATOR. Souza et al. - 0 principal atrativo deste estudo é a apresentação de um criativo simulador de PEEPi, permitindo então o estudo das alterações de mecânica ventilatória causadas por diferentes níveis de PEEPi mesmo em voluntários sadios. O bservou-se aumento do trabalho respiratório e da amplitude da variação da pressão esofágica quando foi simulada a presença de PEEPi de $10 \mathrm{cmH}_{2} \mathrm{O}$ (valor encontrado com freqüência na prática clínica). 0 uso de valores crescentes de CPAP $\left(2-10 \mathrm{cmH}_{2} \mathrm{O}\right)$ administrados por uma máscara facial foi capaz de atenuar as alterações da mecânica ventilatória causadas pela presença do PEEPi, reproduzindo os efeitos benéficos do uso de CPAP não invasivo em pacientes com hiperinsuflação dinâmica (ex.: insuficiência ventilatória em DPOC agudizada). Apoio: LIM-HC/FMUSP, PRONEX.

DUAL-CONTROL MODES NONINVASIVE VENTILATION: PERFORMANCE CHARACTERISTICS OF VAPSV AND VOLUME SUPPORT (VS) VENTILATION IN A NONINVASIVE RESPIRATORY SYSTEM MODEL. Schettino et al. - Experimento realizado em um simulador do sistema respiratório desenvolvido especialmente para o estudo da mecânica ventilatória durante a VNI - máscara facial fixada à cabeça de um manequim, via aérea do manequim conectada a um modelo mecânico dos pulmões. Diferentes condições de impedância e esforços inspiratórios podem ser simulados. Os resultados demonstram que o desempenho do modo VAPSV (volume corrente garantido com fluxo inspiratório livre) foi superior ao do modo VS nos parâmetros analisados (volume corrente efetivo, trabalho inspiratório, aceleração do fluxo inspiratório). Este estudo deixa claro que as limitações do modo VS (valor da pressão de suporte ajustada a cada ciclo para atingir o VT predeterminado) tornam-se mais evidentes na presença de vazamento. Apoio: FAPESP, LIM-HC/FMUSP, PRONEX.

\section{REFERÊNCIAS}

1. A mato MBP, Barbas CSV, Medeiros DM, et al. Effect of a protective-ventilation strategy on mortality in the acute respiratory distress syndrome. $\mathrm{N}$ Engl J Med 1998;338:347-354.

2. A shbaugh DG, Bigelow DB, Petty $T L$, Levine BE. Acute respiratory distress in adults. Lancet 1967;2:319-323.

3. Bernard G, et al. The A merican-European Consensus Conference on ARDS Am J Respir Crit Care Med 1994;149:818-824.

4. Tutor JD, Mason CM, Dobard E, Beckerman RC, Summer WR, Nelson S. Loss of compartmentalization of alveolar tumor necrosis factor after lung injury. Am J Respir Crit Care Med 1994;149:1107-1111.

5. Roy CST, Mizer LA, Kindt GC, Weisbrode SE, Moore SA, Dorinsky PM. Acid aspiration-induced acute lung injury causes leukocyte-dependent systemic organ injury. J Appl Physiol 1993;74:1994-2003.

6. Gullo A, Berlot $G$, Viviani $M$. The role of adult respiratory distress syndrome in the multiple organ dysfunction syndrome. Acta Anaesthesiol Scand 1996; 109:70-73.

7. Pugin J, Verghese G, Widmer MC, Matthay MA. The alveolar space is the site of intense inflammatory and profibrotic reactions in the early phase of acute respiratory distress syndrome. Am J Respir Crit Care Med 1999;27: 304-312.
8. Imai Y, Kawano T, Miyasaka K, Takata M, Imai T, O kuyama K. Inflammatory chemical mediators during conventional ventilation and during high frequency oscillatory ventilation. Am J Respir Crit Care Med 1994;150:1550-1554.

9. Takata M, A be J, Tanaka H, Kitano Y, Doi S, Kohsaka T, Miyasaka K. Intraalveolar expression of tumor necrosis factor- $\alpha$ gene during conventional and high frequency ventilation. Am J Respir C rit Care Med 1997;156:272-279.

10. Von Bethmann AN, Brasch F, Nusing R, Vogt K, Volk HD, Muller KM, Wendel A, U hlig $S$. Hyperventilation induces release of cytokines from perfused mouse lung. Am J Respir Crit Care Med 1988;157:263-272.

11. Tremblay L, Valenza LF, Ribeiro SP, Li J, Slutsky AS. Injurious ventilatory strategies increase cytokines and c-fos m-RNA expression in an isolated rat lung model. J Clin Invest 1997;99:944-952.

12. Dreyfuss $D$, Saumon $G$. Ventilator-induced lung injury: lessons from experimental studies. Am J Respir Crit Care Med 1998;157:294-323.

13. Chiumello D, Pristine G, Slutsky AS. Mechanical ventilation affects local and systemic cytokines in an animal model of acute respiratory distress syndrome. Am J Respir Crit Care Med 1999;160:109-116.

14. Slutsky AS. ACCP Consensus Conference. Mechanical Ventilation. Chest 1993;104:1833-1859.

15. Pierson D. Alveolar rupture during mechanical ventilation: role of PEEP, peak airway pressure and distending volume. Respir Care 1988;33:472-486.

16. Parker J C, Hernandez LA, Peevy KJ . Mechanisms of ventilator-induced lung injury. Crit Care Med 1993;21:131-143.

17. Hida W, Hildebrandt J . Alveolar surface tension, lung inflation, and hydration affect interstitial pressure [Px(f)]. J Appl Physiol 1984;57:262-270.

18. Mellins RB, Levine OR, Skalak R, Fishman AP. Interstitial pressure of the lung. Circ Res 1969;24:197-212.

19. Miserocchi G, Negrini D, Gonano C. Direct measurement of interstitial pressure in in situ lung with intact pleural space. J A ppl Physiol 1990;69:21682174.

20. Sasaki H, Hoppin FC, J r., Takishima T. Peribronchial pressure in excised dog lungs. J A ppl Physiol 1978;45:858-869.

21. Snyder JV, Froese A. Respirator lung. In: Snyder JV, Pinsky MR, eds. Oxygen Transport in the Critically III. Chicago: Year Book Medical Publishers, Inc., 1987;358-373.

22. Albert RK, Lakshminarayan S, Kirk W, Butler J. Lung inflation can cause pulmonary edema in zone I of in situ dog lungs. J Appl Physiol 1980;49: 815-819.

23. Bshouty Z, Younes M. Effect of breathing pattern and level of ventilation on pulmonary fluid filtration in dog lung. Am Rev Respir Dis 1992;145:372376.

24. Costello ML, Mathieu-Costello O, West J B. Stress failure of alveolar epithelial cells studied by scanning electron microscopy. Am Rev Respir Dis 1992; 145:1446-1455.

25. Elliott AR, Fu Z, Tsukimoto K, Prediletto R, Mathieu-Costello O, West J B. Short-term reversibility of ultrastructural changes in pulmonary capillaries caused by stress failure. J Appl Physiol 1992;73:1150-1158.

26. Fu Z, Costello ML, Tsukimoto $K$, et al. High lung volume increases stress failure in pulmonary capillaries. J A ppl Physiol 1992;73:123-133.

27. Mathieu-Costello O, Willford DC, Fu Z, Garden RM, West J B. Pulmonary capillaries are more resistant to stress failure in dogs than in rabbits. J Appl Physiol 1995;79:908-917.

28. West J B, Mathieu-C ostello 0 . Stress failure of pulmonary capillaries: role in lung and heart disease. Lancet 1992;340:762-767.

29. Bshouty Z, Younes M. Effect of tidal volume and PEEP on rate of edema formation in in situ perfused canine lobes. J Appl Physiol 1988;64:19001907.

30. Corbridge TC, Wood LDH, Crawford GP, Chudoba MJ , Yanos J, Sznadjer $\mathrm{J}$ I. Adverse effects of large tidal volume and low PEEP in canine acid aspiration. Am Rev Respir Dis 1990;142:311-315.

31. Dreyfuss D, Saumon G. Role of tidal volume, FRC and end-inspiratory volume in the development of pulmonary edema following mechanical ventilation. Am Rev Respir Dis 1993;148:1194-1203.

32. Dreyfuss D, Saumon G. Should the lung be rested or recruited? The Charybdis and Scylla of ventilator management. Am J Respir Crit Care Med 1994; 149:1066-1068.

33. Dreyfuss $D$, Soler $P, B$ asset $G$, Saumon G. High inflation pressure pulmonary edema. Respective effects of high airway pressure, high tidal volume 
and positive end-expiratory pressure. Am Rev Respir Dis 1988;137:11591164.

34. Hernandez LA, Peevy KJ , Moise AA, Parker J C. Chest wall restriction limits high airway pressure-induced lung injury in young rabbits. J Appl Physio 1989;66:2364-2368.

35. Mascheroni D, Kolobow T, Fumagalli R, Moretti MP, Buckhold D. Acute respiratory failure following pharmacologically induced hyperventilation: an experimental study. Intensive Care Med 1988;15:8-14.

36. Muscedere J G, Mullen J BM, Slutsky AS. Tidal ventilation at low airway pressures can augment lung injury. Am J Respir Crit Care Med 1994;149:13271334.

37. Tsuno K, Miura K, Takeya M, Kolobow T, Morioka T. Histopathologic pulmonary changes from mechanical ventilation at high peak airway pressures. Am Rev Respir Dis 1991;143:1115-1120.

38. Webb HH, Tierney DF. Experimental pulmonary edema due to intermittent positive pressure ventilation with high inflation pressures: protection by positive end-expiratory pressure. Am Rev Respir Dis 1974;110:556-565.

39. McCulloch PR, Forkert PG, Froese AB. Lung volume maintenance prevents lung injury during high frequency oscillatory ventilation in surfactant-deficient rabbits. Am Rev Respir Dis 1988;137:1185-1192.

40. Argiras EP, Blakeley CR, Dunnill MS, Otremski S, Sykes MK. High PEEP decreases hyaline membrane formation in surfactant deficient lungs. $\mathrm{Br}$ J Anaesth 1987;59:1278-1285.

41. Gerstmann DR, DeLemos RA, Coalson JJ, et al. Influence of ventilatory technique on pulmonary baroinjury in baboons with hyaline membrane disease. Pediatr Pulmonol 1988;5:82-91.

42. Macklem PT, Murphy B. The forces applied to the lung in health and disease. Am J Med 1974;57:371-377.

43. Mead J , Takishima T, Leith D. Stress distribution in lungs: a model of pulmonary elasticity. J Appl Physiol 1970;28:596-608.

44. Enhorning G, Robertson B. Lung expansion in the premature rabbit fetus after tracheal deposition of surfactant. Pediatrics 1972;50:58-66

45. Lee $R M, O$ 'Brodovich $H$. A irway epithelial damage in premature infants with respiratory failure. Am Rev Respir Dis 1988;137:450-457.

46. Taghizadeh A, Reynolds EOR. Pathogenesis of bronchopulmonary dysplasia following hyaline membrane disease. Am J Pathol 1976;82:241-264.

47. Goetzman BW. Understanding bronchopulmonary dysplasia. AJ DC 1986; 140:332-334

48. A very ME, Tooley WH, Keller J B, et al. Is chronic lung disease in low birth weight infants preventable? Pediatrics 1987;79:26-30.

49. Rhodes PG, Graves GR, Patel DM, Campbell SB, B lumenthal BI. Minimizing pneumothorax and bronchopulmonary dysplasia in ventilated infants with HMD. J Pedriatr 1983:103:634-637.

50. Van Marter LJ , Pagano M, Allred EN, Leviton A, Kuban KCK. Rate of bronchopulmonary dysplasia as a function of neonatal intensive care practices. J Pediatr 1992;120:938-946.

51. A mato MBP, Barbas CSV, Medeiros D, et al. Beneficial effects of the "open lung approach" with low distending pressures in ARDS: a prospective randomized study on mechanical ventilation. Am J Respir Crit Care Med 1995; 152:1835-1846

52. Dreyfuss $D$, Saumon $G$. Ventilator-induced lung injury: lessons from experimental studies. Am J Respir Crit Care Med 1998;157:294-323.

53. Grunauer MA, A mato MBP, Valente CSB, et al. The lower inflection point of the static PxV curve (Pflex) may not predict "complete alveolar opening" in normal lungs. Am J Respir Crit Care Med 1997;ALA/ ATS:A505.

54. Lachmann B. Open the lung and keep the lung open. Intensive Care Med 1992;18:319-321.

55. Verbrugge S J C, J ong J W, Keijzer E, Anda GV, Lachmann B. Purine in bronchoalveolar lavage fluid as a marker of ventilation-induced lung injury. Crit Care Med 1999;27:779-783.

56. Pesenti A, Fumagali R. PEEP: blood gas cosmetics or a therapy for ARDS? Crit Care Med 1999:27:253-254.

57. Schettino G. Ventilação não invasiva com volume garantido na pressão de suporte (VAPSV): Estudo dos parâmetros ventilatórios em um simulador mecânico. Tese doutorado. Faculdade de Medicina da U niversidade de São Paulo, 1998;119 p.

58. Schettino G, Souza R, Barbas C, Amato M, Carvalho C. Noninvasive VAPSV: Effects of preset parameters and mask fit on the ventilator trigger. Am J Respir Crit Care Med 1998;157:A686.
59. Schettino G, Souza R, Barbas C, A mato M, Carvalho C. Noninvasive VAPSV: Effects of preset parameters and mask fit on the ventilator trigger. Am J Respir Crit Care Med 1998;157:A686.

60. Gay P, Hess D, Hollets S, et al. A randomized prospective trial of noninvasive proportional assist ventilation vs pressure support ventilation to treat acute respiratory insufficiency. Am J Respir Crit Med 1999;159:A14.

61. Vitacca M, Rubini F, Foglio K, Scalvini S, Nava S, A mbrosino N. Noninvasive modalities of positive pressure ventilation improve to the outcome of acute exacerbations in COLD patients. Intensive Care Med 1993;19:450 455

62. Appendini L, Patessio A, Zanaboni S, et al. Physiologic effects of positive en-expiratory pressure and mask pressure support during exacerbations of chronic obstructive pulmonary disease. Am J Respir Crit Care Med 1994 149:1069-1076.

63. Girault C, Richard J C, Chevron V, et al. Comparative physiologic effects of noninvasive assist-control ventilation in acute hypercapnic respiratory failure. Chest 1997;111:1639-1648

64. Elliott MW, Aquilina R, Green M, Moxham J, Simonds A. A comparison of different modes of noninvasive ventilatory support: effects on ventilation and inspiratory muscle effort. A naesthesia 1994:49:279-283.

65. Mehta S, J ay GD, Woolard RH, et al. Randomized, prospective trial of bilevel versus continuous positive airway pressure in acute pulmonary edema. Crit Care Med 1997;25:620-628

66. Calderini E, Confalonieri M, Puccio PG, Francavilla N, Stella L, Gregoretti C. Patient-ventilator asynchrony during noninvasive ventilation: the role of expiratory trigger. Intensive Care Med 1999:25:662-667.

MORPHOLOGICAL CHANGES OF CAROTID BODIES IN ACUTE RESPIRATORY INSUFFICIENCY: A LIGHT MICROSCOPY MORPHOMETRIC STUDY IN HUMANS.

Vinhaes ENG, Dolhnikoff M, Saldiva PHN. Dept. of Pathology, São Paulo, SP, Brazil.

The carotid bodies are structures considered as chemoreceptors sensible to hypoxia. The morphological alterations that this organ shows after chronic hypoxia (high altitudes residents, chronic obstructive pulmonary disease - COPD) and arterial hypertension are well known. In these clinical situations there are also changes in the respiratory control, mainly the response to acute hypoxia. In this study we performed a quantitative analysis of the histology of carotid bodies collected from autopsies of 4 groups of patients: acute respiratory failure (acute respiratory distress syndrome - ARDS) ( $n=7)$, systemic arterial hypertension $(n=7)$, COPD $(n=8)$ and controls $(n=9)$. U sing morphometry, we measured the volume proportion of the 4 main histological cell subtypes found within the glomic tissue: light, dark, sustentacular and progenitor cells. We found a significant increase in the volume proportion of dark cells in the ARDS group when compared to the other groups $(0.22 \pm 0.05 ; 0.11 \pm 0.04 ; 0.09 \pm 0.03 ; 0.12 \pm 0.05$, respectively) $(p<0.0001)$. Patients with COPD showed a significant increase in sustentacular cells and reduced glomic tissue when compared to controls $(p<0.03)$. These results suggest that chronic and acute hypoxia have different effects on the histology of the glomic tissue. These findings are compatible with an acute response of the carotid bodies to hypoxia and indicate the necessity of functional studies of respiratory control in patients surviving ARDS.

\section{PRESSURE-VOLUME CURVES IN ARDS: COMPARISON OF TWO} METHODS.

Meyer EC, Gaudêncio AMAS, Salge J M, O kamoto VN, Grunauer MA, Carvalho CRR, A mato MBP, Barbas CSV. Respiratory ICU-U niversity of São Paulo, SP, Brazil.

PEEP and tidal volume titration, according to the pressure volume (P-V) curves improved survival in ARDS patients. There are severa techniques described to study P-V curves, however, there are few studies comparing them. In order to determine the correlation between 
two different techniques of acquisition P-V curves we compared the lower (Lpflex) and upper inflection point (Upflex) of the static occlusion technique (SOC) versus the constant flow technique (CF) in 13 ARDS patients (LIS > 2.5). Volume variation was monitored by an inductive body an inductive body plethysmograph and by a flow transducer. Random VTs were tested after a fixed lung volume history. End inspiratory pressure after a $2 \mathrm{~s}$ pause was recorded. CF was performed with a $1 \mathrm{~L}$ / min flow after a $40 \mathrm{cmH}_{2} \mathrm{O}$ CPAP maneuver followed by disconnection. To prevent bias, a mathematical algorithm was used for Pflex calculation. A regression line of points corresponding to the $80 \%$ of maximal slope segment (Slope ${ }_{\max }$ ) was calculated. Regression lines of the points below and above Slope $\max _{\max }$ were also calculated. $L P_{\text {flex }}$ and $U P_{\text {flex }}$ were identified as the intersection of the regression lines. Results: $L P_{\text {flex }}$ and $U P_{\text {flex }}$ could be identified in all patients in the CF technique, while $L P_{\text {flex }}$ was identified in 12 patients, and $U P_{\text {flex }}$ in only 9 patients in the SOC technique. $L P_{\text {flex }}$ and $U P_{\text {flex }}$ are higher in the $C F$ technique $\left(\mathrm{LP}_{\text {flex }}\right.$ was $16.0 \pm 1.4$ and $13.6 \pm 1.5 ; \cup \mathrm{P}_{\text {flex }}$ was $33.1 \pm 1.6$ and $27.6 \pm 1.6$, respectively for $\mathrm{CF}$ and SOC). The track of SOC P-V curves is shifted to the left when compared with CF. Conclusion: $P_{\text {flex }}$ could be identified in the majority of the patients. The difference observed between the two methods could be attributed to different lung time history and recruitment during SOC procedure.

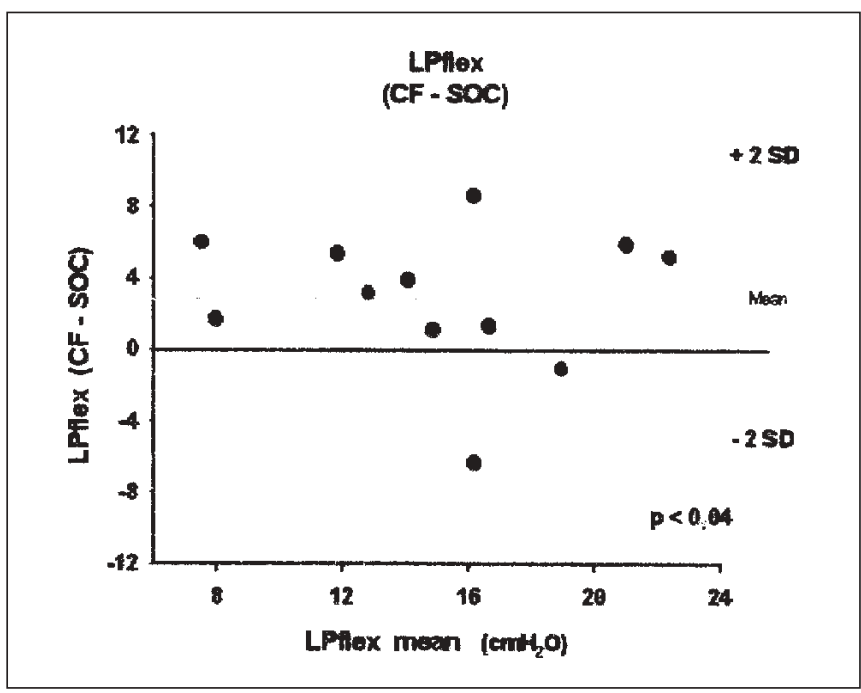

TIDAL RECRUITMENT MISINTERPRETED AS VISCOELASTIC PRESSURE DISSIPATION IN ARDS PATIENTS.

Grunauer MA, Barbas CSV, Meyer EC, Alzugaray PF, Carvalho CRR, A mato MBP. Respiratory ICU, Pulmonary Division, University of São Paulo, SP, Brazil.

Increased "lung tissue resistance" or viscoelastic pressure dissipation in patients with ARDS has been well documented. We suspected that part of this altered viscoelastic behavior could be caused by tidal recruitment (accommodation of tidal volume into new units). The purpose of this study is to analyze the viscoelastic dissipation of pressures across the respiratory system at progressive lung recruitment/ de-recruitment conditions. We studied six ARDS patients, $<5$ days of evolution, LIS > 2.5). Incremental and decremental PEEP steps $\left(2 \mathrm{cmH}_{2} \mathrm{O}\right.$ up to $P_{\text {PLAT }} 55 \mathrm{cmH}_{2} \mathrm{O}, V_{\mathrm{T}}=4 \mathrm{~mL} / \mathrm{kg}$, inspiratory pause $=2 \mathrm{~s}$ ), were subsequently applied and $\mathrm{P}(\mathrm{P}=$ plateau pressure subtracted from pressure at zero flow) was calculated at each PEEP level. Lung volume changes were monitored with a body plethysmograph. Results: besides the non-linearity of pressure dissipations along different PEEP levels (fig. 1) a significant difference of $P$ between the incremental and decremental PEEP steps was also observed ( $p<0.0001$ ). Conclusions: 1) A linear model describing the non Newtonian component of lung tissue resistance is not valid. 2) P is strongly influenced by lung recruitment. 3) "Classic" stress relaxation and stress recovery may depend on major changes of lung surface area during inspiratory pause.

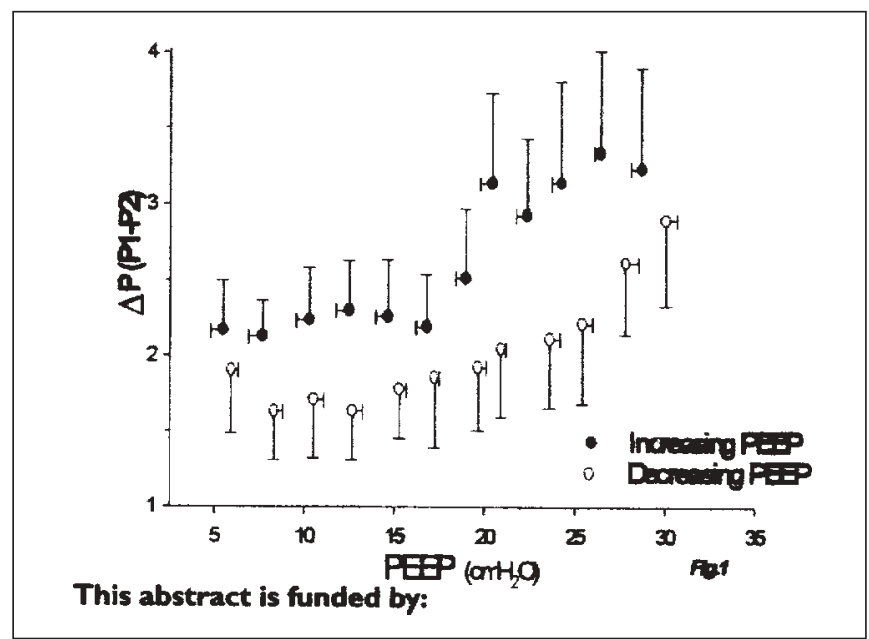

DIFFERENT CONSTANT FLOWS CAN EQUALLY DETERMINE THE LOWER INFLECTION POINT IN ARDS PATIENTS.

Gama AMCN, Meyer EC, Gaudêncio AMSA, Grunauer MA, A mato MBP, Carvalho CRR, Barbas CSV. Respiratory ICU-U niversity of São Paulo, SP, Brazil.

The titration of the ideal PEEP by the lower inflection point of the $P x V$ curve of the respiratory system $\left(L-P_{\text {flex }}\right)$ associated with low tidal ventilation led to an improvement in survival in ARDS patients. A mong the possible techniques to obtain the PxV curve at the beside the low constant flow (CF) is the easiest and quickest one. In order to evaluate the influence of 4 different $C F(1,2,5$ and $10 \mathrm{~L} / \mathrm{min})$ in the determination of $\mathrm{L}-\mathrm{P}_{\text {flex }}$ and in inspiratory work $\left(\mathrm{Wi}-\mathrm{L}_{\mathrm{xcmH2O}}-\right.$ till $1.35 \mathrm{~L}$ above the FRC) we studied 7 ARDS patients (LIS $>2.5,<5$ days of evolution). After a CPAP maneuver of $40 \mathrm{cmH}_{2} \mathrm{O}$ per $15 \mathrm{~s}$ and 10 s exhalation to atmosphere, the 7 patients randomly received the 4 inspiratory $C F$. We analyzed the PxV curve time, $L-P_{\text {flex }}$ and Wi among the different flows, estimating the volume from the CF at ATPS (VCF) comparing with the measured volume by the respiratory inductive plethysmograph (RIP).

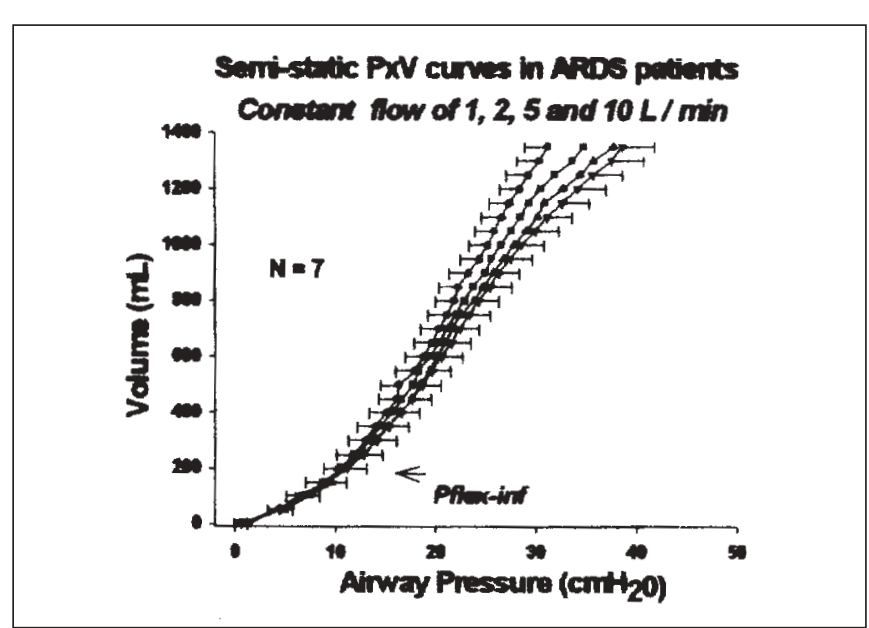




\begin{tabular}{cccccc}
\hline $\begin{array}{c}\mathbf{N}=\mathbf{7} \\
\text { FLO W }\end{array}$ & T (s) & Wi (VCF) & Wi (RIP) & $\begin{array}{c}\mathbf{L - P f}_{\text {lex }} \\
\mathbf{c m H}_{\mathbf{2}} \mathbf{0}\end{array}$ & L-P $_{\text {flex }}$ (RIP) \\
$1 \mathrm{~L} / \mathrm{min}$ & $74 \pm 4$ & $25 \pm 2$ & $27 \pm 2$ & $11.4 \pm 4$ & $11.7 \pm 4$ \\
$2 \mathrm{~L} / \mathrm{min}$ & $39 \pm 4$ & $27 \pm 2$ & $26 \pm 1$ & $12.2 \pm 4$ & $12.0 \pm 4$ \\
$5 \mathrm{~L} / \mathrm{min}$ & $17 \pm 1$ & $28 \pm 2$ & $27 \pm 2$ & $12.0 \pm 4$ & $12.4 \pm 5$ \\
$10 \mathrm{~L} / \mathrm{min}$ & $9 \pm 0.3$ & $28 \pm 1$ & $28 \pm 2$ & $12.3 \pm 4$ & $12.1 \pm 4$ \\
\hline
\end{tabular}

There was no difference of the L- $P_{\text {flex }}$ or Wi among the different CFs comparing the estimated or measured volume. Conclusion: CF from 1 to $10 \mathrm{~L} / \mathrm{min}$ can equally determine the $L-P_{\text {flex }}$ in ARDS patients.

PEEP AFTER A RECRUITMENT MANEUVER CAN KEEP A GREATER MEAN ALVEOLAR DIAMETER IN VALI RATS.

Mori SS, Dolhnikoff M, Gaudêncio AMAS, A mato MBP, Martins MA, Barbas CSV. Respiratory ICU and Department of pathology - University of São Paulo, SP, Brazil.

The effects of PEEP on lung morphometry before and after a recruitment maneuver according to the PxV curve in ventilator associated lung injury (VALI) rats is not well known. We studied 48 wistar rats $(290 \mathrm{~g})$ randomly assigned to eight groups after 3 semi-static PV curves with a constant flow of $1.66 \mathrm{~mL} / \mathrm{s}$ were done. Groups: 1 - Fixation at CPAP of zero, 2 - Fixation at CPAP of $30 \mathrm{cmH}_{2} \mathrm{O}, 3$ - Fixation at CPAP of zero after VALI, 4 - Fixation at CPAP of $30 \mathrm{cmH}_{2} \mathrm{O}$ after VALI, 5 - Fixation at CPAP $2 \mathrm{cmH}_{2} \mathrm{O}$ below L-P-Flex after VALI, 6 Fixation at CPAP $2 \mathrm{cmH}_{2} \mathrm{O}$ above the L-P-Flex after VALI, 7 - Fixation at CPAP $2 \mathrm{CmH}_{2} \mathrm{O}$ below L-P-Flex after a recruitment maneuver of CPAP $=30 \mathrm{cmH}_{2} \mathrm{O}$ after VALI, 8 - Fixation at CPAP $2 \mathrm{cmH}_{2} \mathrm{O}$ above L-P-Flex after CPAP $=30 \mathrm{cmH}_{2} \mathrm{O}$ after VALI. VALI was induced ventilating the rats with a $V_{T}$ of $45 / \mathrm{mL} / \mathrm{kg}$ with $5 \mathrm{cmH}_{2} \mathrm{O}$ of PEEP until observing a increment of $30 \%$ of the respiratory system elastance. $U$ sing morphometry we measured the mean linear intercept $(L \mathrm{~m})$, perivascular edema index $(\mathrm{Ei})$, hemorrhage index $(\mathrm{Hi})$ and inflammation index (li). Results:

\begin{tabular}{ccclcl}
\hline $\mathbf{G}$ & $\mathbf{C P A P ~} \mathbf{~ C m H}_{2} \mathbf{O}$ & $\mathbf{E i}$ & $\mathbf{H i}$ & $\mathbf{I i}$ & $\mathbf{L m}(\boldsymbol{\mu m})$ \\
$\mathbf{N = 8}$ & & & & & \\
1 & 0 & $1.0 \pm 7$ & $2 \pm 2$ & $0 \pm 0$ & $42 \pm 9$ \\
2 & 30 & $0.7 \pm 2$ & $1 \pm 1$ & $0 \pm 0$ & $59 \pm 22$ \\
3 & 0 & $1.3 \pm 2$ & $5 \pm 2^{*}$ & $2 \pm 1$ & $38 \pm 11$ \\
4 & 30 & $1.4 \pm 4$ & $4 \pm 0^{*}$ & $1 \pm 0$ & $55 \pm 16$ \\
5 & $17 \pm 1$ & $1.2 \pm 5$ & $4 \pm 1^{*}$ & $2 \pm 1$ & $41 \pm 10$ \\
6 & $22 \pm 1$ & $1.1 \pm 3$ & $4 \pm 2^{*}$ & $1 \pm 0$ & $43 \pm 15$ \\
7 & $20 \pm 1$ & $1.3 \pm 5$ & $3 \pm 1$ & $1 \pm 0$ & $60 \pm 18^{*}$ \\
8 & $22 \pm 1$ & $1.1 \pm 3$ & $3 \pm 1$ & $1 \pm 0$ & $55 \pm 13$ \\
\hline
\end{tabular}

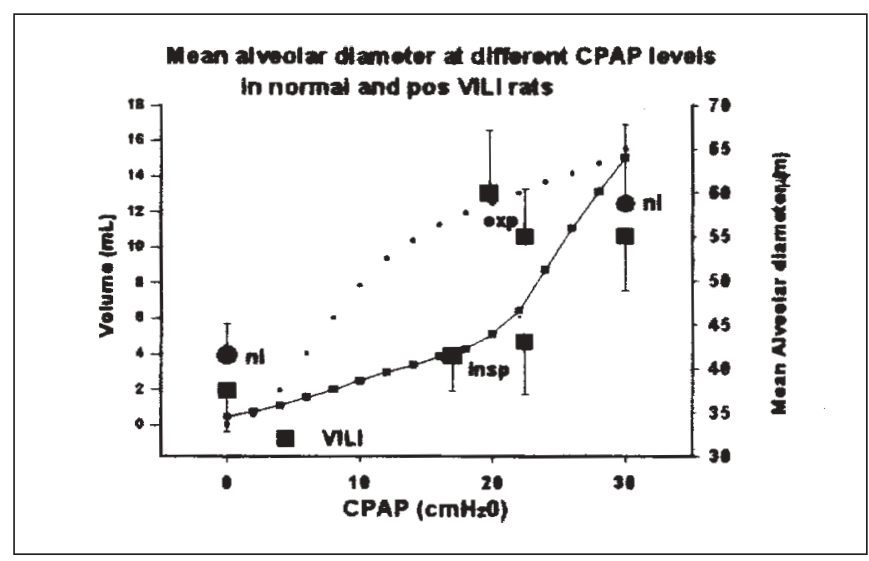

Conclusion: VALI is characterized histological by hemorrhage. Fixation at the same CPAP $2 \mathrm{cmH}_{2} \mathrm{O}$ below the L-Pflex after a recruitment maneuver leads to a greater mean alveolar diameter in VALI rats.

FINDINGS THE "BEST PEEP" IN ARDS PATIENTS INFLUENCE OF TIDAL VOLUME AND LUNG RECRUITMENT IN THE ESTIMATION OF THE BEST COMPLIANCE.

Meyer EC, Barbas CSV, Grunauer MA, Caramez MP, Souza R, Carvalho MEP, Carvalho CRR, A mato MBP. Respiratory ICU, U niversity of São Paulo, SP, Brazil.

The correct adjust of PEEP and tidal volume $\left(\mathrm{V}_{T}\right)$ can protect patients with ARDS from ventilator induced lung injury (VILI). "B est PEEP" could be set according to the best compliance, but this approach could be influenced by $V_{T}$ and lung recruitment. We studied 6 ARDS patients (mean LIS $=3,2$ ) in order to determine the influence of $V_{T}$ and lung recruitment (at the PEEP level) on the static compliance (Cst, rs). We did a step-by-step ascending and descending maneuver with high $\left(\mathrm{HV}_{\mathrm{T}}\right)$ and low $V_{T}\left(L V_{T}\right) ; 10$ and $4 \mathrm{~mL} / \mathrm{kg}$, respectively. PEEP steps of 2 $\mathrm{CmH}_{2} \mathrm{O}$ was applied up to a $\mathrm{P}_{\text {PLAT }} 55 \mathrm{cmH}_{2} \mathrm{O}$, and than it was removed. Volume variation was monitored with an inductive body plethysmograph. Respiratory work during ascending (W, rs, asc) and descending phases (W, $\mathrm{rs}$, desc) were calculated on the area of the PEEP-end expiratory lung volume (EEL V) curve. Best PEEP was obtained from PEEP/ Cst, rs curve W, rs, asc was higher than W, rs, desc $\left(14.0 \pm 1.1\right.$ versus $9.1 \pm 1.5 \mathrm{~L} / \mathrm{cmH}_{2} \mathrm{O}$ for $\mathrm{LV}_{\mathrm{T}}$ and $11.9 \pm 0.8$ versus $8.9 \pm 1.1 \mathrm{~L} / \mathrm{cmH}_{2} \mathrm{O}$ for $\mathrm{HV}_{\mathrm{T}} ; \mathrm{p}<0.03$ in both comparisons). The $\mathrm{W}$, $r s$, asc of $\mathrm{HV}_{\mathrm{T}}$ was lower than the $\mathrm{W}$, rs, asc of $\mathrm{LV}_{\mathrm{T}}(\mathrm{p}<0.05)$, but both $W, r s$, desc were not different. In spite of the lower $W, r s$, asc of $H_{T}$, LVt reached a higher level of lung volume $(2.0$ versus $1.5 \mathrm{~L})$. Best PEEP was higher in $\mathrm{LV}_{\mathrm{T}}$ than in $\mathrm{HV}_{\mathrm{T}}(12.3 \pm 2.0$ versus $6.6 \pm 1.6$ $\left.\mathrm{CmH}_{2} \mathrm{O}, \mathrm{p}<0.05\right)$. Best PEEP in the descending phase was higher than in the ascending phase for both $\mathrm{LV}_{\mathrm{T}}$ and $\mathrm{HV}_{\mathrm{T}}$. Conclusion: $H \mathrm{~V}_{T}$ can cause tidal recruitment which is responsible for the higher $\mathrm{Cst}$, rs and the lower $\mathrm{W}, \mathrm{rs}$, asc, however, the Lvt reached a higher lung recruitment at the same pressure limit, influencing the calculation of the best PEEP. After a high pressure recruiting maneuver a given PEEP value could achieve a higher EELV.

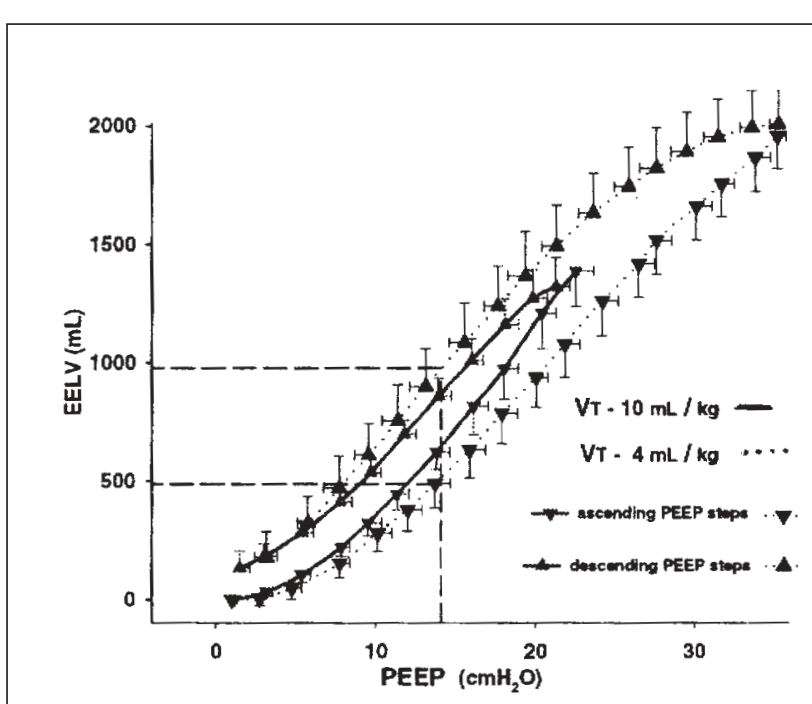

This abstract is funded by: 
DISTRIBUTION OF OPENING AND CLOSING PRESSURES IN ARDS PATIENTS DERIVED FROM THE PRESSURE-VOLUME (PV) RELATIONSHIP.

Venegas J G, Harris RS, Amato M. Massachusetts General Hospital and Harvard Medical School, Boston, MA and Respiratory ICU, University of São Paulo, SP, Brazil.

A previously reported empirical equation fitted animal and human PV data extremely well. In that equation, the function: $F=\left(1+e^{(c-P) / d}\right)$, approximates the integral of a normal distribution and could represent the progressive recruitment or de-recruitment of alveoli with normally distributed opening or closing pressures, respectively. We multiplied such a "fractional recruitment" function by the classic exponential "elastic function" yielding: $V=b\left(1-e^{-k P}\right) /\left(1+e^{(c-P)} / d\right)$, where $b, k, c$, and $d$ correspond to vital capacity, elastic coefficient, mean opening or closing pressure, and a distribution width (proportional to the standard deviation), respectively. We fitted inspiratory and expiratory PV curves from eight ARDS patients obtained with the super-syringe method up to pressures of $55 \mathrm{cmH}_{2} \mathrm{O}$. The new equation fitted the data with high accuracy $\left(R_{2}=0.9996\right)$ yielding an average mean closing pressure " $c$ " that was $7 \mathrm{cmH}_{2} \mathrm{O}$ lower than the mean opening pressure. The values of deflation " $\mathrm{k}$ " were all within the normal range, thus supporting the "baby lung" concept in ARDS.

\begin{tabular}{lcccc}
\hline & $\mathbf{b}(\mathbf{m l})$ & $\mathbf{k}\left(\mathbf{c m H}_{\mathbf{2}} \mathbf{O}^{-1}\right)$ & $\mathbf{c}\left(\mathbf{c m H}_{\mathbf{2}} \mathbf{0}\right)$ & $\mathbf{d}\left(\mathbf{c m H}_{\mathbf{2}} \mathbf{0}\right)$ \\
Inflation & 2298 & 0.034 & 17.3 & 6.1 \\
Deflation & 1920 & 0.080 & 10.3 & 7.3 \\
\hline
\end{tabular}

EFFECTS OF FUROSEMIDE IN RESPIRATORY MUCUS PROPERTIES AND TRANSPORTABILITY IN PATIENTS UNDER MECHANICAL VENTILATION.

Kondo CS, Macchione M, Guimarães ET, Carvalho CRR, King M, Saldiva PH N, Lorenzi-Filho G. Pulmonary Division and Department of Pathology, School of Medicine, University of São Paulo and Federal University of São Paulo, SP, Brazil and Pulmonary Research Group, University of Alberta, Edmonton, Canada.

The use of IV furosemide is common practice in patients under mechanical ventilation (MV), however its effects on the respiratory mucus are largely unknown. Furosemide can affect the respiratory mucus either directly through inhibition of $\mathrm{NaK}(\mathrm{Cl})_{2}$ co-transporter on the basolateral surface of airway epithelium or indirectly through increased diuresis and patient's dehydration. We investigated the mucus physical properties and transportability of mucus obtained from 27 patients under MV distributed in 2 groups, furosemide (10-200 mg IV) $(n=12)$ and control $(n=15)$. Mucus was collected at time $0,1,2,3$ and 4 hours. Mucus was studied by means of a micrortheometer, in vitro mucus transport (MT) (frog palate), contact angle (CA) and cough clearance (CC) (simulated cough machine). MT remained constant in the control group and decreased significantly in the furosemide group $1.01 \pm 0.21,0.81 \pm 0.16 *, 0.77 \pm 0.20 *, 0.82 \pm 0.22 *$ and $0.82 \pm$ $0.20 *$ at time $0,1,2,3$ and 4 hs, respectively $(* p<0.001$, different from 0 ). The remaining parameters did not change significantly in both groups. We conclude that IV furosemide can acutely impair MT in patients under MV.

FLOW PATTERNS IN NORMAL SUBJECTS UNDER PRESSURE SUPPORT VENTILATION AT DIFFERENT SLOPES AND INSPIRATORY RESISTANCE.

Borges J B, Pompilio CE, Barbas CSV, A mato MBP. Respiratory ICU and University Hospital, University of São Paulo, SP, Brazil.

Background: Pressure support ventilation (PSV) is a ventilatory mode which theoretically permits a total control of inspiratory time (Ti) by the patients. But, $\mathrm{Ti}$ is strongly dependent on peak flow, ventilator end inspiratory criteria, patient respiratory drive, and initial flow rate (pressure slope). Objective: analyze the flow pattern at different flow rates with normal and high airway resistance. Methods: we studied 3 normal male subjects under PSV by a facial mask delivered by a Evita 2 ventilator (Dräger, Lubeck - Germany) that allows a fine set of initial flow rate. The pressure (PS) was preset to achieve a tidal volume (VT) of nearly $10 \mathrm{~mL} / \mathrm{kg}$ (mean $\left.8 \mathrm{cmH}_{2} \mathrm{O}\right)$ at zero PEEP with a $\mathrm{FiO}_{2}$ enough to maintain a $\mathrm{SatO}_{2}$ above $96 \%$ (mean 0.3 ). The flow rate was changed from $0.064 \mathrm{~s}$ to $0.75 \mathrm{~s}$ and $1.5 \mathrm{~s}$. Then, a external resistance of 20 $\mathrm{cmH}_{2} \mathrm{O} / \mathrm{l} / \mathrm{s}$ was connected to the airways. The following variables were recorded and analyzed: $\mathrm{Ti}$, inspiratory peak flow (PF), time to achieve peak flow $\left(T_{P F}\right)$ and $V T$. Results: the PF and $T_{P F}$ changed significantly with flow rates ( $p=0.04$ and $p<0.001$, respectively) at the normal resistance procedure. At the high resistance, only the $T_{P F}$ changed significantly $(p<0.001)$. Both VT and Ti did not vary in the two procedures.

\begin{tabular}{lrrrcrcrc}
\hline SLOPE & 0.064 & \multicolumn{1}{c}{0.75} & 1.5 & $P$ & 0.064 & 0.75 & 1.5 & $P$ \\
RESIST & NORMAL & NORMAL & NORMAL & HIGH & & \multicolumn{1}{c}{ HIGH } & \multicolumn{1}{c}{ HIGH } & \\
TPF(s) & $0.14 \pm 0.0$ & $0.89 \pm 0.0$ & $1.36 \pm 0.1$ & $<0.001$ & $0.097 \pm 0.0$ & $0.9 \pm 0.1$ & $1.47 \pm 0.1$ & $<0.001$ \\
PF (L/S) & $1.027 \pm 0.2$ & $0.759 \pm 0.1$ & $0.619 \pm 0.1$ & $<0.05$ & $0.57 \pm 0.0$ & $0.52 \pm 0.0$ & $0.495 \pm 0.1$ & $=0.09$ \\
VT & $1.04 \pm 0.2$ & $1.14 \pm 0.1$ & $1.03 \pm 0.1$ & $=0.71$ & $0.9 \pm 0.1$ & $0.89 \pm 0.2$ & $0.95 \pm 0.1$ & $=0.92$ \\
Ti & $1.82 \pm 0.2$ & $2.29 \pm 0.4$ & $2.33 \pm 0.2$ & $=0.20$ & $2.29 \pm 0.3$ & $2.39 \pm 0.4$ & $2.44 \pm 0.4$ & $=0.92$ \\
\hline
\end{tabular}

Conclusion: The VT and Ti remained unchanged with different flow rates and normal and high resistance. These findings suggested that VT and Ti could be the most important determinants of flow pattern in normal subjects under PSV in different slopes and inspiratory resistance conditions.

\section{VENTILATORY MECHANICS PATTERNS DURING THE USE OF AN} INTRINSIC PEEP SIMULATOR.

Souza R, Schettino G, Salge J M, Kuwakino MH, A mato MBP, CarvaIho CRR. Respiratory ICU, University of São Paulo, SP, Brazil.

Background: the presence of intrinsic PEEP (PEEPi) has major implications on the respiratory mechanics of patients with obstructive airway disease. We do not know any model of PEEPi simulator that allows the study of PEEPi and its ventilatory mechanics effects in healthy humans. Objective: evaluate the ventilatory mechanics changes with an original PEEPi simulator. Material and Method: we developed a PEEPi simulator using a facial mask connected to a $Y$ circuit where a compressive latex tube in a water column was added to the expiratory limb (positive pressure in circuit $=P E E P$ ) and an unidirectional valve to the inspiratory lim, in order to allow the start of the inspiratory flow only after the pressure in the mask becomes lower than the ambient pressure. In this system we named the pressure at the mask and at the

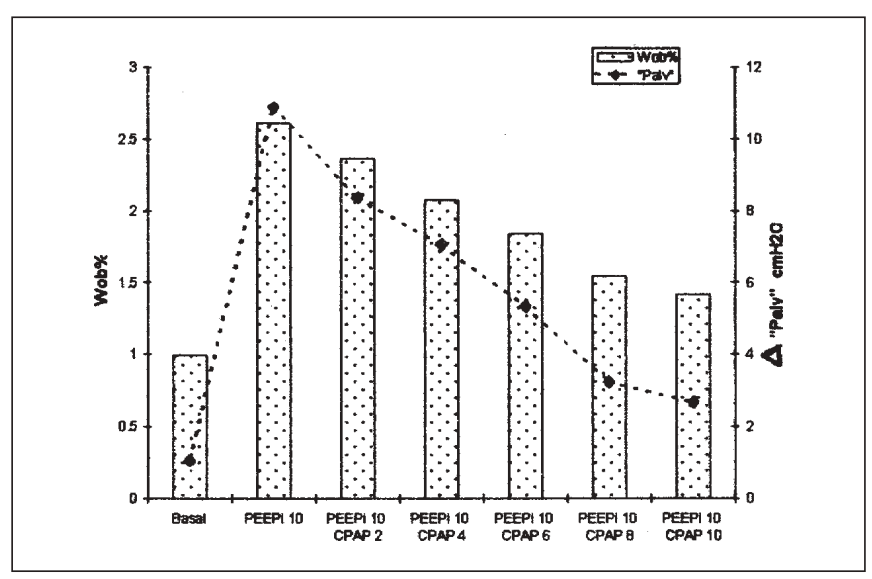


circuit as "alveolar" pressure ("Palv"); and the pressure proximal to the unidirectional valve as "airway" pressure ("Paw"). We studied 5 healthy volunteers under spontaneous ventilation. The ventilatory mechanics were obtained at basal conditions (no PEEPi); with PEEPi $=10 \mathrm{cmH}_{2} \mathrm{O}$; with PEEPi $=10 \mathrm{cmH}_{2} \mathrm{O}$ and progressive CPAP levels to increase the "Paw". The analyzed parameters were: 1 ) work of breathing, showed as the relation between work obtained in each step and work in the basal condition (Wob\%); 2) "Palv" variation ("Palv") showed as the necessary pressure variation in the circuit to allow the beginning of the inspiratory flow. Results: the presence of PEEPi resulted in an increase in W\% and "Palv". The use of CPAP was effective in attenuate the W\% and "Palv" due to PEEPi - Figure. Conclusions: the simulator was effective in the study of ventilatory mechanics pattern due to the presence of PEEPi.

DUAL-CONTROL MODES NONINVASIVE VENTILATION: PERFORMANCE CHARACTERISTICS OF VAPSV AND VOLUME SUPPORT (VS) VENTILATION IN A NONINVASIVE RESPIRATORY SYSTEM MODEL.

Schettino G, Souza R, Barbas CSV, Amato MBP, Carvalho CRR. Respiratory ICU, University of São Paulo, SP, Brazil.

Background: the ability of VAPSV and VS to assure a preset VT and decrease the work of breathing during noninvasive positive pressure ventilation (NPPV) is not well known. O bjective: evaluate the ventilatory mechanics of VAPSV and VS, also comparing with volume assist-control (Vac), during NPPV. Material and Method: we used a stimulator of respiratory system consisted of a head model where a pneumatic face mask was fitted. The head airway was connected to a mechanical lung model. Different conditions of compliance $(C)$, resistant $(\mathrm{R})$ and inspiratory efforts ( $\mathrm{E}-\mathrm{L} / \mathrm{min}$ ) were simulated and ventilated by the studied modes. The ventilators were used as following: BIRD $8400 \AA$ Vac - VT $740 \mathrm{ml}$, inspiratory square flow (IF) IL/ S VAPSV VT $740 \mathrm{ml}$, IF IL/s, PS $\mathrm{cmH}_{2}$ O. Servo $300 \AA$ VS - VT $740 \mathrm{ml}$, rise time $5 \%$. The analyzed parameters were: 1 ) inspiratory work of breathing (Winsp\%) showed as the relation between Winsp obtained in each simulation and Winsp of $\mathrm{Vac}$ in the basal condition $(\mathrm{C}=85 ; \mathrm{R}=5$; $\mathrm{E}$ $=60) ; 2$ ) VT\% showed as the relation between the VT achieved in the lung model circuit and the preset inspiratory ventilator VT; 3 ) acceleration flow time ( $\mathrm{Ta}-\mathrm{ms}$ ) defined as the time spend to achieved inspiratory flow of $1 \mathrm{~L} / \mathrm{s}$. Results: Winsp \% was smaller in VAPSV than in Vac and VS $(p=0.05$ and $p<0.001)$ and VT\% was similar in the three modes - Figure. Ta was similar in Vac and VAPSV in all simulated conditions ( 45 to $50 \mathrm{~ms})$ and smaller than VS $(70 \mathrm{~ms}-\mathrm{E} 120$ to 220 $\mathrm{ms}-\mathrm{R} 15)$. Significant variations in VT (348 to $720 \mathrm{ml})$ were observed during the use of VS in high) impedance simulation (C 30). Conclusion: the global performance of VAPSV is superior to VS in experimental conditions of NPPV.

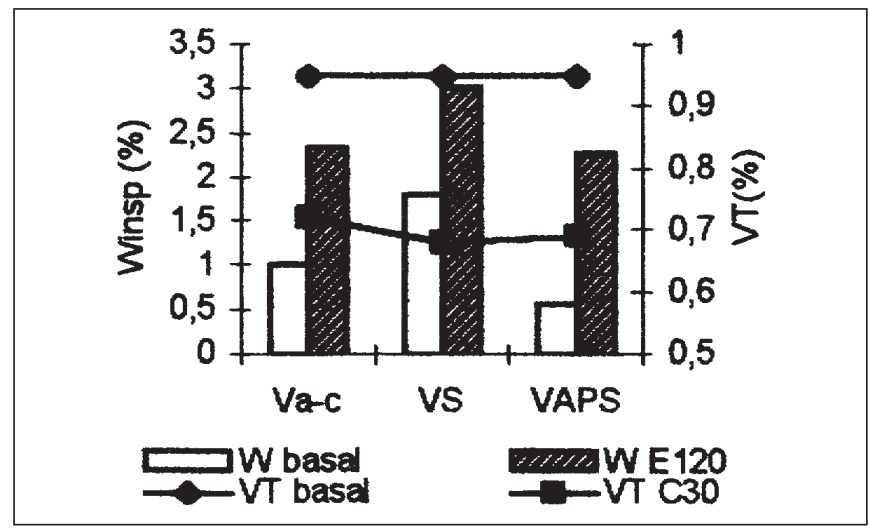

REPETITIVE HIGH PRESSURE RECRUITMENT MANEUVERS (RM) REQUIRED TO MAXIMALLY RECRUIT LUNG IN ARDS SHEEP MODEL.

Fujino Y, Goddon S, Hess D, A mato MBP, Kacmarek RM. A nesthesia and Respiratory Care, Massachusetts General Hospital and Harvard Medical School, Boston MA and Pulmonary Division, Hospital das Clínicas - University of São Paulo, SP, Brazil.

Lung recruitment in ARDS occurs at peak inspiratory pressures. We compared the effectiveness of the two maneuvers designed to maximally recruit the lung. Method: saline lavage injured sheep were randomly assigned to 3 groups: CPAP $40 \mathrm{cmH}_{2} \mathrm{O}$ for 1 min (CPAP40) $(n=3)$ pressure controlled ventilation $20 \mathrm{cmH}_{2} \mathrm{O}$ with PEEP $40 \mathrm{cmH}_{2} \mathrm{O}$ for 2 min (PCV60) ( $n=3$ ) and no RM (control) $(n=3)$. A fter each RM animals were ventilated with $P C V$, rate $20 / \mathrm{min}, \mathrm{I}: \mathrm{E} 1: 1$, peak airway pressure $35 \mathrm{cmH}_{2} \mathrm{O}$ and PEEP $20 \mathrm{cmH}_{2} \mathrm{O}$ for 30 min. A nimals were then disconnected from the ventilator for 30 seconds followed by another RM and 30 min ventilation. Each RM was repeated 4 times. Results: all animals survived the study period. Oxygenation improved significantly $(p<0.05)$ in the PCV 60 group after the second RM. There was a nonsignificant tendency for further recruitment with additional Rms. Lung tissue is being histologically evaluated for extent of lung injury.

\begin{tabular}{|c|c|c|c|c|c|}
\hline & \multicolumn{5}{|c|}{$\mathrm{PaO}_{2}(\mathrm{mmH} \mathrm{g})$ at injury and following each RM } \\
\hline & INJURY & RM 1 & RM2 & RM3 & RM4 \\
\hline $\begin{array}{l}\text { Control } \\
\text { CPAP40 } \\
\text { PCV60 }\end{array}$ & $\begin{array}{l}82 \pm 13 \\
77 \pm 12 \\
96\end{array}$ & $\begin{array}{r}163 \pm 87 \\
144 \pm 85 \\
99 \pm 17\end{array}$ & $\begin{array}{l}245 \pm 115 \\
198 \pm 121 \\
388 \pm 106^{*}\end{array}$ & $\begin{array}{l}270 \pm 108 \\
220 \pm 136 \\
456 \pm 61 *\end{array}$ & $\begin{array}{l}271 \pm 108 \\
184 \pm 101 \\
473 \pm 23^{2}\end{array}$ \\
\hline
\end{tabular}

Mean \pm SE. Post-hoc $* p<0.05$ vs. Injury

Conclusion: Repetitive RMs with relatively high airway pressure are required to maximally recruit collapsed lung in ARDS.

MANNITOL PARTIALLY PREVENTS ACUTE LUNG INJURY INDUCED BY OLEIC ACID IN RATS.

Hilkner-Silva M, Leme AS, A rantes FM, Lichtenstein A, Saldiva PH N, Martins MA. Department of Medicine, School of Medicine, University of São Paulo, Brazil.

Acute lung injury remains one of the leading causes of death in intensive care units. Infusion of oleic acid $(\mathrm{OA})$ is a classical experimental model of acute lung injury. We studied the effects of infusion of mannitol (MAN, $1 \mathrm{ml}$ of a $20 \%$ solution) on the acute pulmonary changes in OA (100 mg/ $\mathrm{kg})$ infused Wistar male rats. We infused MAN 15 minutes before or 2 hours after $O A$ infusion. We studied four groups of rats: a) $O A+M A N$, b) $O A$ + saline $(O A+S A L)$, c) $S A L+M A N$, d) SAL + SAL. We measured the amount of alveolar edema and respiratory unevenness (alveolar collapse + hyperdistention) 4 hours after infusion of $\mathrm{OA}$ by morphometric analysis. Respiratory unevenness index (means \pm SEM):

\begin{tabular}{|c|c|c|c|c|c|c|c|}
\hline \multicolumn{4}{|c|}{ MAN 15 minutes before $A O$} & \multicolumn{4}{|c|}{ MAN 2 hours after $A 0$} \\
\hline $\begin{array}{l}S A L+S A L \\
(n=14)\end{array}$ & $\begin{array}{c}\text { MAN + SAL } \\
(n=12)\end{array}$ & $\begin{array}{r}S A L+A O \\
(n=16)\end{array}$ & $\begin{array}{c}M A N+A O \\
(n=16)\end{array}$ & $\begin{array}{c}S A L+S A L \\
(n=16)\end{array}$ & $\begin{array}{c}S A L+\text { MAN } \\
(n=13)\end{array}$ & $\begin{array}{c}O A+S A L \\
(n=16)\end{array}$ & $\begin{array}{c}O A+M A N \\
(n=14)\end{array}$ \\
\hline $20.5 \pm 1.2$ & $23.0 \pm 1.6$ & $27.6 \pm 1.4 *$ & $22.1 \pm 1.0$ & $19.5 \pm 0.7$ & $19.4 \pm 1.0$ & $26.2 \pm 0.8 *$ & $18.4 \pm 0.8$ \\
\hline
\end{tabular}

${ }^{*} p<0.05$ compared to other three groups

Alveolar Edema Index (means \pm SEM):

\begin{tabular}{|c|c|c|c|c|c|c|c|}
\hline \multicolumn{4}{|c|}{ MAN 15 minutes before AO } & \multicolumn{4}{|c|}{ MAN 2 hours after AO } \\
\hline $\begin{array}{l}S A L+S A L \\
(n=14)\end{array}$ & $\begin{array}{c}\text { MAN + SAL } \\
(n=12)\end{array}$ & $\begin{array}{l}S A L+A O \\
(n=16)\end{array}$ & $\begin{array}{c}\text { MAN + AO } \\
(n=16)\end{array}$ & $\begin{array}{c}S A L+S A L \\
(n=16)\end{array}$ & $\begin{array}{c}S A L+\text { MAN } \\
(n=14)\end{array}$ & $\begin{array}{l}O A+5 A L \\
(n=16)\end{array}$ & $\begin{array}{c}O A+\text { MAN } \\
(n=14)\end{array}$ \\
\hline $8.5 \pm 1.2$ & $10.7 \pm 3.5$ & $18.9 \pm 3.8$ & $10.2 \pm 1.9$ & $7.7 \pm 1.5$ & $7.4 \pm 1.3$ & $17.2 \pm 4.7$ & $7.3 \pm 1.5$ \\
\hline
\end{tabular}


We conclude that morphometric analysis is a good method for detecting pulmonary lesions in acute lung injury induced by oleic acid as early as 4 hours after infusion of $\mathrm{OA}$. Mannitol infusion partially prevents this acute lung injury.

EXTRACELLULAR MATRIX (ECM) REMODELING AND LUNG MECHANICS IN EARLY AND LATE ARDS: DIFFERENCES BETWEEN PULMONARY AND EXTRA-PULMONARY DISEASE.

Negri EM, H oelz C, Barbas CSV, Montes GS, Saldiva PH N, Capelozz VL. Department of Pathology, Hospital das Clínicas and Instituto do Coração, University of São Paulo, School of Medicine, São Paulo, Brazil.

In order to evaluate the alterations in ECM in the acute respiratory distress syndrome, 37 ARDS patients, mean age $46 \pm 13$ years old, were retrospectively studied and divided in groups according to the phase (early or late disease) and to the etiologic of ARDS: pulmonary (i.e., pneumonia) or extra-pulmonary (i.e., sepsis, extracorporeal circulation). U sing the image analysis, the collagen and elastic fibers content of the alveolar septum were quantified in autopsied lung samples, stained by the picrossirius and the oxidated resorcin fucsin methods respectively. In addition, values of dynamic compliance of the respiratory system at the day of death were retrospectively accessed. Data were analyzed by ANOVA, setting the significance in $5 \%$. In the early phase of ARDS collagen fibers content was significantly higher in the pulmonary group, no differences were observed in fibers content in the late phase of the disease between groups. Codyn was always higher in extra-pulmonary ARDS in the early and late phases. We conclude that pulmonary remodeling in ARDS seems to be influenced by the initial site of injury (pulmonary or extra-pulmonary) and mechanical properties of lung parenchyma may be influenced by the differences.
OXYGENATION AND MECHANICS PROFILE BEFORE AND AFTER CARDIOPULMONARY BYPASS.

Crespo AS, Zin WA, Carvalho AF, Silveira C, Campos LA, Dohmann HJ . Pró-Cardíaco Hospital - Postoperative ICU - Anesthesia Department, RJ , Brazil.

Introduction: Cardiopulmonary bypass (CPB) is a well known factor that potentially decreases exposure of blood to non-endotelial surfaces, ischemia/ reperfusion of organs, protamine reactions and others. The lung, by its physiological particularities, is one of the main organs affected by the capillary leak syndrome, leading to oxygenation and mechanics changes. This study aims to investigate the immediate alterations in lung function after CPB in adult patients during cardiac surgeries. Method: Twenty four patients of both sexes (age: 36-82 y; weight: $56-92 \mathrm{~kg}$ ) were studied before and after CPB. General anesthesia was induced and maintained with fentanyl, midazolan and low concentration of isoflurane. Respiratory monitoring was achieved by means of intermittent measurements of arterial blood gases and continuous evaluation of $\mathrm{PETCO}_{2}$, inspired fraction of oxygen $\left(\mathrm{FIO}_{2}\right)$ and expired tidal volumes $\left(V_{T}\right)$ flows, pressures, static compliance (Cst) and resistance (Raw) by a solid state/ single beam nondispersal infrared unit main stream capnography with fixed orifice differential pressure pneumotach ( $\mathrm{CO}_{2}$ SMPLUS-Novametrics). Data were gathered before and after $\mathrm{CPB}$, and analyzed by Kolmogorov-Smirnov test, modified by Lilliefors. Comparison between groups were performed by Student's $t$ test and Mann-Whitney rank sum test. Significance was set at $5 \%$. Results: CPB time varied from 60 to $185 \mathrm{~min}$ (mean $=108.3$ ). Data base $\mathrm{CPB}$ was $\mathrm{PaO}_{2} / \mathrm{FIO}_{2} 417.52 \pm 124.15$. Cst $52.04 \pm 18.43$ and raw of $12.25 \pm 5.95$. After $\mathrm{CPB}$ the $\mathrm{PaO}_{2} / \mathrm{FIO}_{2}$ was $320.08 \pm 100.71$; Cst $49.21 \pm 17.07$ and raw of $12.08 \pm 5.29$. Conclusion: in patients with the same respiratory pattern, considering both pre and post CPB, we found a significant decrease in oxygenation parameters, not followed by significant changes in lung mechanics as would be expected. 